\title{
From pre-storm activity to magnetic storms: a transition described in terms of fractal dynamics
}

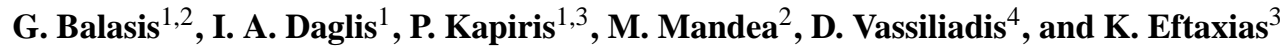 \\ ${ }^{1}$ Institute for Space Applications and Remote Sensing, National Observatory of Athens, Metaxa and Vas. Pavlou St., Penteli, \\ 15236 Athens, Greece \\ ${ }^{2}$ GeoForschungsZentrum Potsdam, Telegrafenberg, 14473 Potsdam, Germany \\ ${ }^{3}$ Solid State Section, Physics Department, University of Athens, Panepistimiopolis, Zografos, 15784 Athens, Greece \\ ${ }^{4}$ NASA Goddard Space Flight Center, Greenbelt, Maryland 20771, USA
}

Received: 18 December 2005 - Revised: 21 November 2006 - Accepted: 24 November 2006 - Published: 21 December 2006

\begin{abstract}
We show that distinct changes in scaling parameters of the $D_{s t}$ index time series occur as an intense magnetic storm approaches, revealing a gradual reduction in complexity. The remarkable acceleration of energy release - manifested in the increase in susceptibility - couples to the transition from anti-persistent (negative feedback) to persistent (positive feedback) behavior and indicates that the occurence of an intense magnetic storm is imminent. The main driver of the $D_{s t}$ index, the $V B_{\text {South }}$ electric field component, does not reveal a similar transition to persistency prior to the storm. This indicates that while the magnetosphere is mostly driven by the solar wind the critical feature of persistency in the magnetosphere is the result of a combination of solar wind and internal magnetospheric activity rather than solar wind variations alone. Our results suggest that the development of an intense magnetic storm can be studied in terms of "intermittent criticality" that is of a more general character than the classical self-organized criticality phenomena, implying the predictability of the magnetosphere.
\end{abstract}

Keywords. Magnetospheric physics (Magnetospheric configuration and dynamics; Solar wind-magnetosphere interactions; Storms and substorms)

\section{Introduction}

Magnetic storms (MSs) occur when the accumulated input power from the solar wind exceeds a certain threshold. MSs are a main element of space weather: they have severe impacts on both space-borne and ground-based technological systems (Daglis et al., 2001). MS intensity is usually represented by an average of the geomagnetic perturbations measured at four mid-latitude magnetic observatories, known as the $D_{s t}$ index (http://swdcwww.kugi.kyoto-u.ac.jp/). Intense

Correspondence to: G. Balasis

(gbalasis@space.noa.gr)
MSs are relatively rare events; as in the case of tropospheric storms, earthquakes, solar flares, etc., the occurrence of geomagnetic storms rapidly decreases as their magnitude grows. The present study is initially focused on one year of $D_{s t}$ data (2001), characterized by two important events, in the first and last trimesters of the considered interval.

The complex system of the Earth's magnetosphere, which corresponds to an open spatially extended nonequilibrium (input - output) system, manifests itself in linkages between space and time, producing characteristic fractal structures (Freeman and Watkins, 2002). In this paper, the fractal spectral properties of the $D_{s t}$ data are examined using wavelet analysis methods developed by Torrence and Compo (1998), Balasis et al. (2005) and Mandea and Balasis (2006). We point out that the same wavelet tools have been applied by Mandea and Balasis (2006) to satellite magnetic data for an astrophysical application, thus showing remarkable applicability to the delineation of fine electromagnetic structures contained within geophysical signals (http://www. sciencemag.org/content/vol314/issue5798/twil.dtl). Here, it is shown that distinct changes in associated scaling parameters emerge as large magnetospheric disturbances approach (e.g. 31 March 2001 and 6 November 2001 with minimums $D_{s t}-387 \mathrm{nT}$ and $-292 \mathrm{nT}$, respectively).

Since the magnetospheric activity is determined to a large extent by variations in the interplanetary medium, it is important to differentiate between scalings produced by the variability of the interplanetary magnetic field and those due to internal processes. Therefore, we have applied similar tools of analysis to corresponding time series of the solar wind driver variables, $V B_{\text {South }}$. Our findings suggest that the transition in the $D_{s t}$ scaling represents the effects of internal magnetospheric processes rather than interplanetary variations. Our conclusions are supported with results from another solar cycle maximum (1989).

Published by Copernicus GmbH on behalf of the European Geosciences Union. 


\section{Fractal spectral analysis of the $D_{s t}$ time series}

Observations suggest that under the influence of the solar wind, the magnetosphere can be channelled into a globally non-equilibrium critical state (Chang, 1999; Consolini and Chang, 2001, 2002). A common hallmark of out-ofequilibrium phenomena is their extraordinary complexity. Complex systems self-organize their internal structure and their dynamics, showing novel and surprising macroscopic properties, including coherent large-scale collective behaviors. A universal footprint seen in many complex systems near criticality is the self-affinity for energy release from the system that signals a fractal topology, namely a multi-scale process with no preferred spatial and temporal scales. The fractional power-law relationship is a standard definition of a self-affine structure.

A basic reason for our interest in complexity is the striking similarity in behavior near the critical point among systems that are otherwise quite different in nature (Stanley, 1999, 2000; Viczek, 2001, 2002; Sornette, 2004). Lots of work on complexity focuses on power-law distributions which describe the scaling properties of fractal processes and structures (e.g. Malamud et al., 1998). Recent studies have demonstrated that a large variety of complex processes, including earthquakes (Bak, 1996), forest fires (Malamud et al., 1998), heartbeats (Peng et al., 1995), human coordination (Gilden et al., 1995), neuronal dynamics (Worrell et al., 2002) and financial markets (Mandelbrot, 2005; Kiyono et al., 2006) exhibit statistical similarities, most commonly power-law scaling behaviors of a particular observable.

Although self-organized criticality (SOC) can exist, strictly speaking, only in the limit of infinitely slow external input, where a complete separation of time scales is achieved (Gedalin et al., 2005), it has also been applied to presumably strong driving avalanching systems. A good example of such systems is space plasma and, in particular, the plasma in the Earth's magnetotail under magnetic substorm conditions (Chang et al., 2006). Additionally, Jun and Wu (2005) have recently found a large-scale intermittency in a system which is strongly driven in turbulence by an external source.

If a time series is a temporal fractal, then a power-law of the form

$S(f) \propto f^{-\beta}$

is obeyed, with $S(f)$ the power spectral density and $f$ the frequency. The spectral scaling exponent $\beta$ is a measure of the strength of time correlations. The suitability of the fit of a time series to the power-law is represented by the linear correlation coefficient, $r$, of this representation.

A way to examine transient phenomena is to divide the measurements into time windows and analyze these windows. If this analysis yields different results for some precursory time intervals, then a transient behavior can be extracted. Thus, we first examine whether the underlying complexity manifests itself in linkages between space and time, generally producing fractal patterns, as the extreme event is approached. In particular, our attention is directed to whether distinct alterations in the associated scaling parameters emerge when an intense MS approaches. For this purpose, we applied the wavelet analysis technique (Balasis et al., 2005; Mandea and Balasis, 2006) to the $D_{s t}$ time variations, in order to derive the coefficients of its power spectrum.

The wavelet transform provides a representation of the signal in both the time and frequency domains (Kumar and Foufoula-Georgiou, 1997). In contrast to the Fourier transform, which provides the description of the overall regularity of signals, the wavelet transform identifies the temporal evolution of various frequencies (i.e. as a time-frequency plane that indicates the frequency content of a signal at every time). This feature of the wavelet transform is useful for the $D_{s t}$ time series because it is non-stationary and has a timevarying frequency content. The decomposition pattern of the time-frequency plane is predetermined by the choice of the basis function. In the present study, we used the continuous wavelet transform with the Morlet wavelet as the basis function. The results were checked for consistency using the Paul and DOG mother functions (Torrence and Compo, 1998).

The non-stationary character of the $D_{s t}$ index requires methods that can appropriately treat such non-stationarities. In practice, the condition of stationarity for non-stationary signals can be satisfied by dividing the signal into blocks of short, pseudo-stationary segments (Akay, 1997). On the other hand, recent studies show that the wavelet transform can remove effects due to non-stationarities present in the time series (Amaral et al., 1998). For the 1-year period considered here (year 2001), applying the wavelet transform to the hourly $D_{s t}$ values leads to a matrix with $65 \times(365 \times 24)$ elements, where 65 is the number of frequencies. Then, power spectral densities were estimated in the frequency range from 2 to $128 \mathrm{~h}$, using a moving window of $256 \mathrm{sam}-$ ples. The number of samples by which the moving window sections overlap is 255. Finally, the spectral parameters $r$ and $\beta$ were calculated for each window.

In Fig. 1, the $D_{s t}$ time series and its wavelet power spectrum are shown (upper panels), while in Fig. 2, the temporal evolution of its spectral parameters $r$ and $\beta$ are plotted (upper panels). In Figs. 3a-3b, we show examples of the fitting of equation (1) in individual time windows; each of these windows has a duration of $256 \mathrm{~h}$. In Figs. $3 \mathrm{c}-3 \mathrm{~d}$ the error estimates which correspond to the spectra presented in Figs. $3 \mathrm{a}-3 \mathrm{~b}$ are shown.

\subsection{Fractal properties}

The temporal evolution of $r$ (see $r_{D_{s t}}$ plot in Fig. 2) indicates that the fit to the power-law is excellent ( $r$ is always above 0.94). This means that the fractal character of the underlying processes and structures is compact: the activity could be ascribed to a multi-time-scale cooperative activity of numerous 

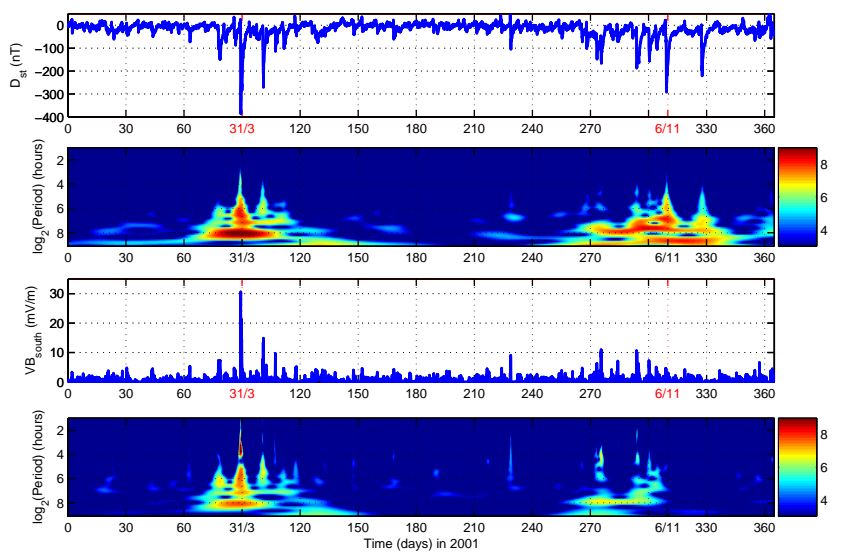

Fig. 1. From top to bottom: $D_{s t}$ time series and its wavelet power spectrum (upper panels); $V B_{\text {South }}$ time series and its wavelet power spectrum (lower panels). The 31 March and 6 November $2001 \mathrm{MSs}$ are marked with red in the time series plots.

activated units, in which an individual unit behavior is dominated by its neighbors, so that all units simultaneously alter their behavior to a common large-scale fractal pattern. In the cases of the 31 March and 6 November 2001 MSs, we observe a further increase in $r$ as the main phase approaches: a region with $r>0.99$ is observed during the last stage of precursory activity. The high $r$-values indicate that the clustering of activated events in more compact fractal structures is strengthened during this period. Such elementary activated events could, in the case of MSs, be successive stages of acceleration and earthward transport of ions - for example due to substorm-induced impulsive electric fields (Daglis et al., 2004). Substorms, as well as regular convection result in multiple ring currents with a distribution of growth/decay times (Liemohn and Kozyra, 2003).

\subsection{The transition from anti-persistent to persistent proper- ties}

Two classes of signal have been widely used to model stochastic fractal time series (Heneghan and McDarby, 2000): fractional Gaussian noise (fGn) and fractional Brownian motion (fBm). These are, respectively, generalizations of white Gaussian noise and Brownian motion. A formal mathematical definition of continuous $\mathrm{fBm}$ was first offered by Mandelbrot and Ness (1968). For the case of the fGn model the scaling exponent $\beta$ lies between -1 and 1 , while the regime of $\mathrm{fBm}$ is indicated by $\beta$ values from 1 to 3 .

For our study, the $\beta$ values (see $\beta_{D_{s t}}$ plot in Fig. 2) are distributed in a region defined from 1 to 3 . This means that the time profile of the $D_{s t}$ time series is qualitatively analogous to $\mathrm{fBm}$, possessing long-range temporal correlations. More precisely, the observed fractal-law $\left(S(f) \propto f^{-\beta}\right)$ indicates the existence of long-term memory. This means that the current value of the geomagnetic signal is correlated not only

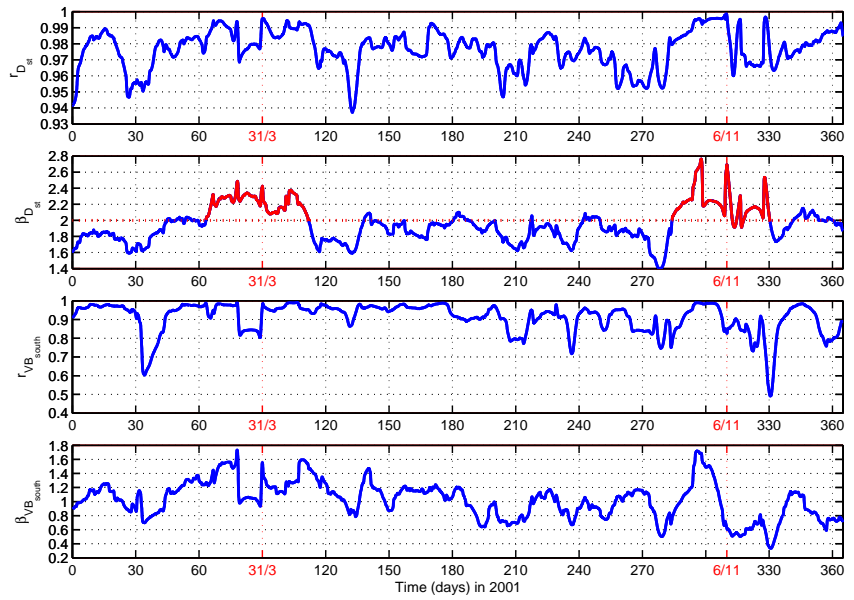

Fig. 2. From top to bottom: $D_{s t}$ linear correlation coefficients $r$ and spectral exponents $\beta$ (upper panels), and corresponding $V B_{\text {South }} r$ and $\beta$ values (lower panels). The spectral parameters were estimated from the wavelet power spectra of Fig. 1 using a 256-hour moving window with an overlap of 255 samples. The red dashed line in $\beta_{D_{s t}}$ plot marks the transition between anti-persistent and persistent behavior.

with its most recent values but also with its long-term history in a scale-invariant, fractal manner. In other words, the system refers to its history in order to determine its future.

The distribution of the $\beta$ exponent is also shifted to higher values as the intense MSs approach. This shift reveals several features of the underlying mechanism. As $\beta$ increases, the spatial correlation in the time series also increases (Turcotte, 1997). This behavior indicates a gradual increase of the memory, and thus a gradual reduction in complexity in the underlying dynamics. This suggests that the occurence of an intense MS may represent a gradual transition from a less orderly state to a more orderly state (see also Sitnov et al., 2001).

The colour type of the power spectrum density $(\beta>0)$ indicates that the spectrum manifests more power at low frequencies than at high frequencies. The spectral exponent $\beta$ is increasing with time, indicating the gradual enhancement of lower frequency fluctuations. This observation is consistent with the following physical picture: the activated partial ring currents coalesce to form larger fractal structures, i.e. the events are initiated at the lowest level of the hierarchy, with the smallest elements merging in turn to form larger and larger ones. This sign may be considered as a precursor of the forthcoming magnetic storm.

The $\beta$ exponent is related to the Hurst exponent, $H$ :

$\beta=2 H+1$

with $0<H<1 \quad(1<\beta<3)$ for the $\mathrm{fBm}$ random field model (Heneghan and McDarby, 2000). The exponent $H$ 


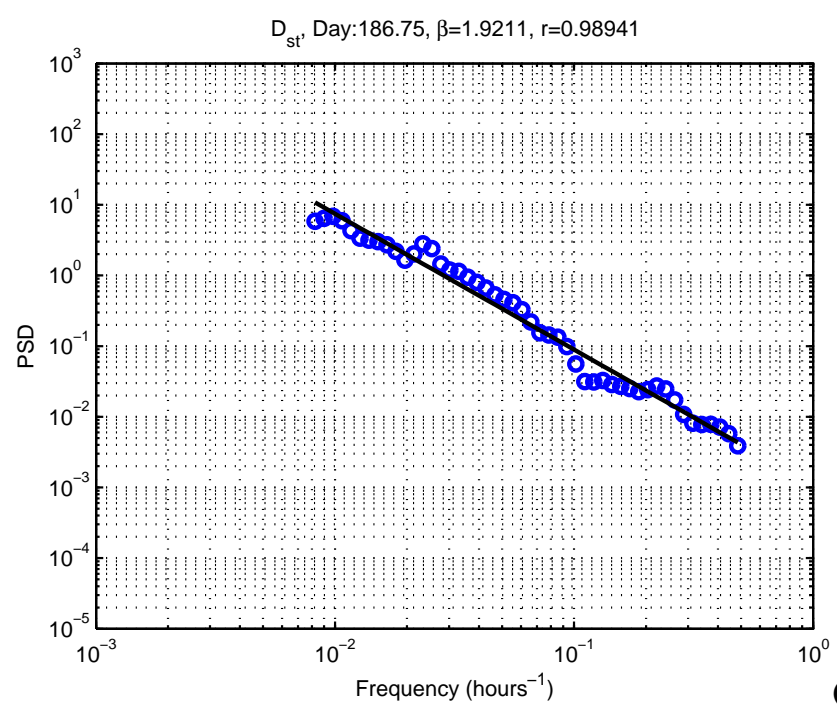

(a)

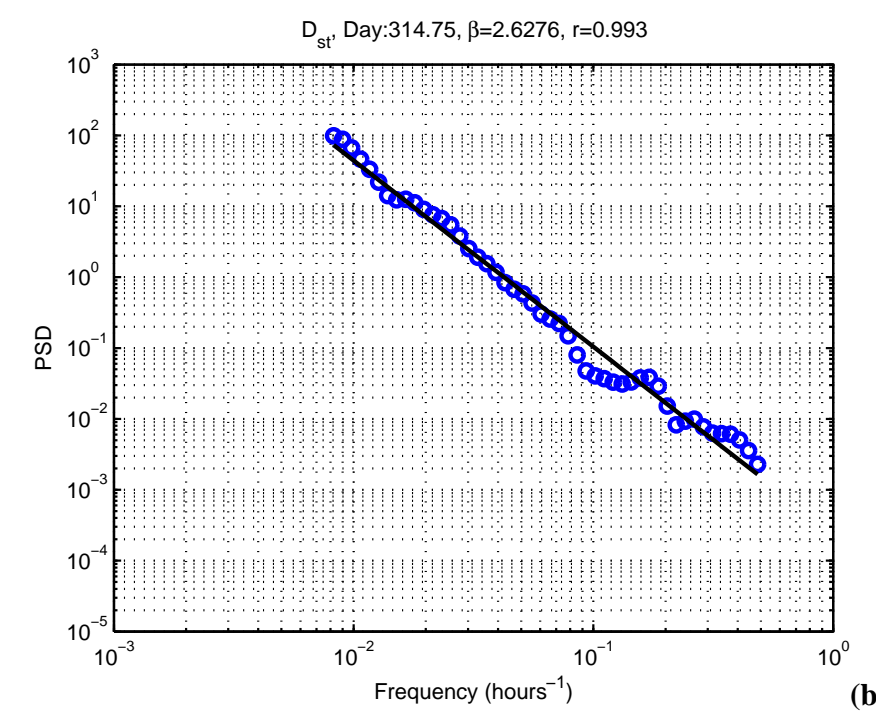

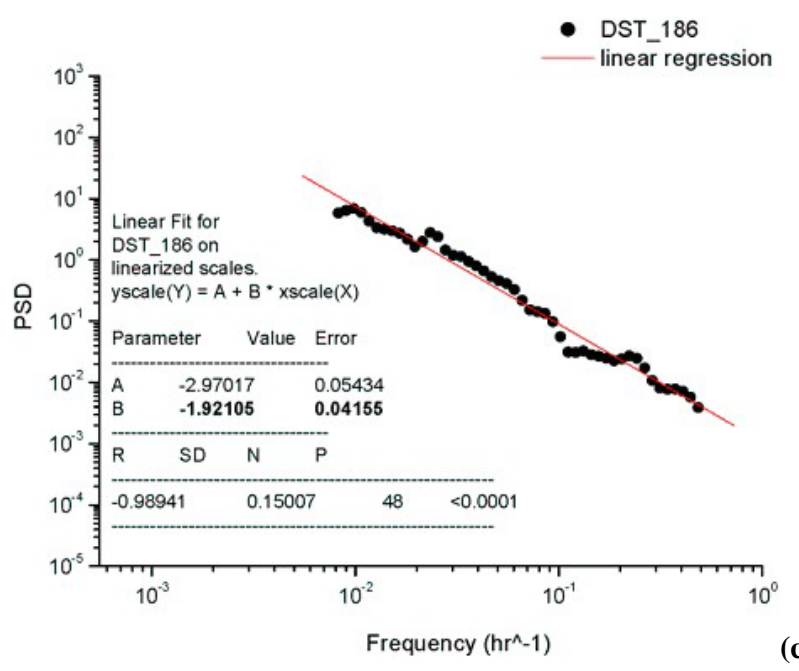

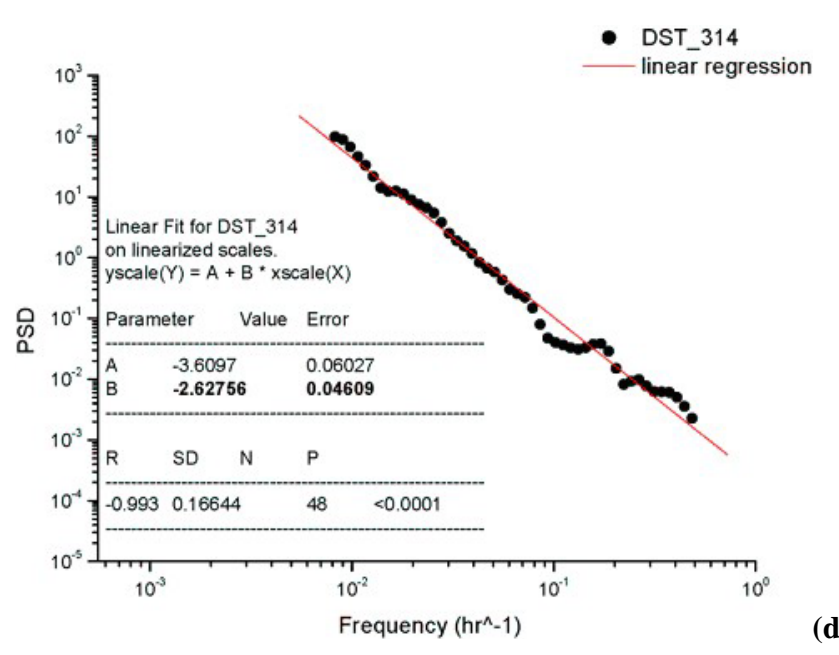

(b)

Fig. 3. (a)-(b) The power spectral density functions of the $D_{s t}$ index for days starting on the values indicated on the top of each diagram and lasting $256 \mathrm{~h}$. The black line gives a fitted power-law of the form $S(f) \propto f^{-\beta}$ to periods 2-128 h. (c)-(d) Error estimates of the linear coefficients $r$ and spectral exponents $\beta$ for the spectra shown in (a) and (b), respectively.

characterizes the persistent/anti-persistent properties of the signal (Kapiris et al., 2003, 2004a, b).

The range $0<H<0.5(1<\beta<2)$ indicates anti-persistency, which means that if the fluctuations increase in a period, it is likely to decrease in the interval immediately following and vice versa. Physically, this implies that fluctuations tend to induce stability within the system (negative feedback mechanism). Figure 2 reveals that the $D_{s t}$ time series exhibits antipersistent properties during the quiet period (i.e. well before and after 31 March and 6 November 2001 MSs).

We draw attention to the fact that $D_{s t}$ exhibits persistent properties, $0.5<H<1(2<\beta<3)$, around 31 March and 6 November $2001 \mathrm{MSs}$ (c.f. Fig. 2: parts of $\beta_{D_{s t}}$ plot, marked in red). This means that if the amplitude of the fluctuations increases in a time interval, it is likely to continue increasing in the immediately next interval. In other words, the underlying dynamics is governed by a positive feedback mechanism. A close up of the $D_{s t}$ time series from days 60 to 120 and 280 to 340, as well as the corresponding wavelet power spectra and spectral parameters $r$ and $\beta$, are given in Figs. 4a and $4 \mathrm{~b}$, respectively.

The value $H=0.5(\beta=2)$ suggests that there is no correlation between the repeated increments. Consequently, this particular value takes on a special physical meaning: it marks the transition between anti-persistent and persistent behavior in the time series. To conclude, one can recognize two different regimes. The first refers to quiet epochs of magnetospheric activity. The associated $D_{s t}$ time series follows 

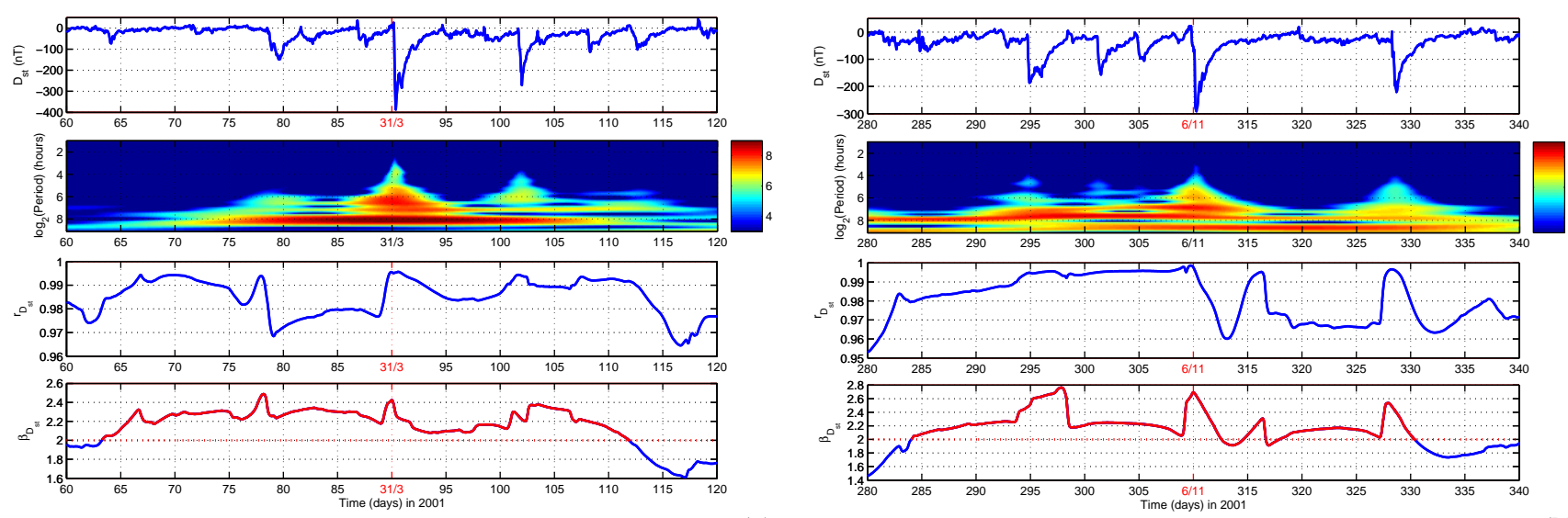

(a)
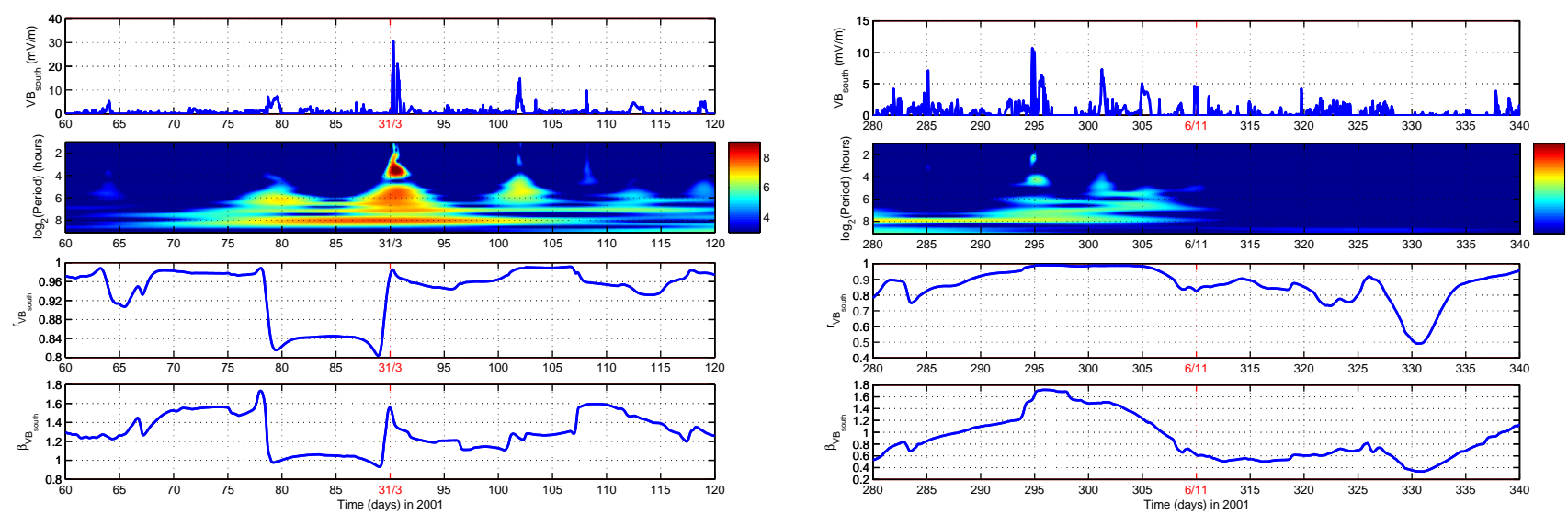

(c)

Fig. 4. (a)-(d) A close-up on days 60 to 120 and 280 to 340 of Figs. 1-2. At each subfigure: $D_{s t}$ or $V B_{\text {South }}$ time series and its wavelet power spectrum (upper panels), corresponding linear correlation coefficients $r$ and spectral exponents $\beta$ (lower panels).

the $\mathrm{fBm}$ model with anti-persistent properties. Figure 5a shows the function $\log S(f)$ versus $f$ for such a quiet period (days 150-240). The anti-persistent behavior is verified. The second regime refers to the epoch including an intense MS. The $D_{s t}$ time series also obeys the fBm model; it, however, shows persistent behavior. Figures $5 \mathrm{~b}-5 \mathrm{c}$ show the function $\log S(f)$ versus $f$ for two such epochs associated with the two intense MSs emerged during the 2001. The plot verifies their persistent behavior. Based on the results of Figs. 5a-5c, Fig. 2 shows the consecutive transitions from one regime to another.

Remark: Figure 5d shows the power spectrum of $D_{s t}$ index for the year 2001. The slope of the spectral line is approximately 2 . This fractal feature suggests that the dynamics of the whole time series is similar to that of a Brownian motion. This result is in harmony with the recent analysis performed by Wei et al. (2004). The average of the power spectral density functions of the $D_{s t}$ index for many years reveals that its power spectrum exhibits some periodicities at frequencies of about 1/(6T), 1/(8T), (1/12T), (1/24T), where $T=1 \mathrm{~h}$, which has also been previously detected by Takalo et al. (1995).

\section{Is the transition in the $D_{s t}$ solely imposed by the solar wind?}

Since the magnetospheric activity is determined to a large extent by variations in the interplanetary medium, it is important to differentiate between scalings produced by the variability of the interplanetary magnetic field and those due to internal processes. Magnetospheric convection, which leads to ring current intensification and an increase in ground geomagnetic disturbances (represented by $D_{s t}$ ), is primarily driven by the solar wind electric field $\mathbf{v} \times \mathbf{B}$, and in particular its negative part, $V B_{\text {South. }}$ In Fig. 1 , the $V B_{\text {South }}$ time series data and its wavelet power spectrum are also shown (lower panels). The correlation coefficient $r$ (see $r_{V} B_{\text {South }}$ plot in Fig. 2) varies from values as low as 0.5 up to almost 


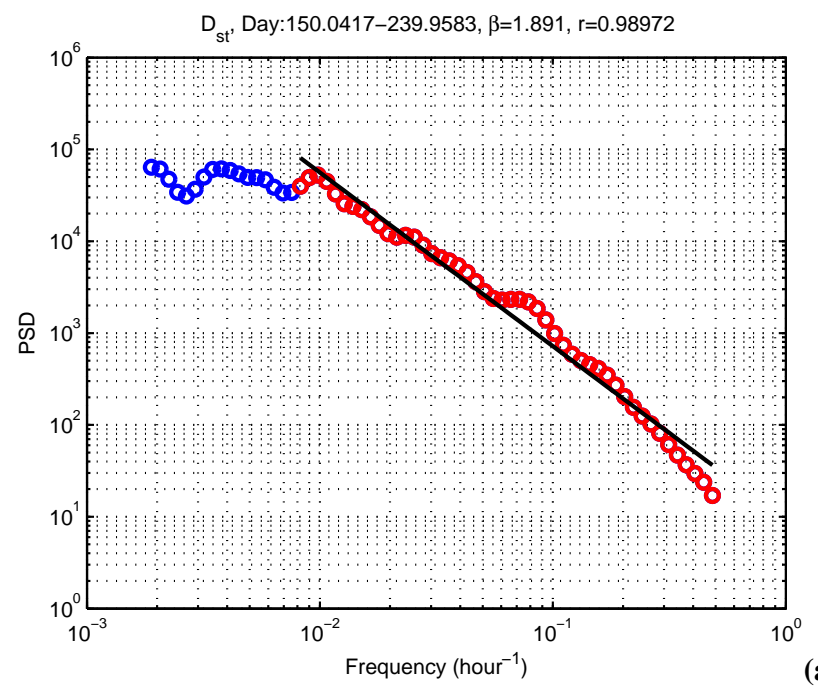

(a)

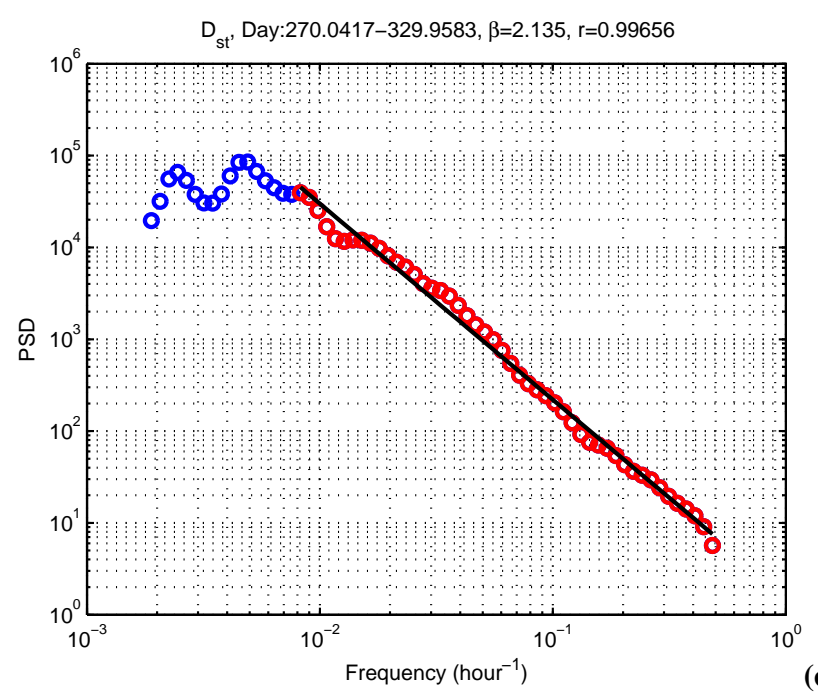

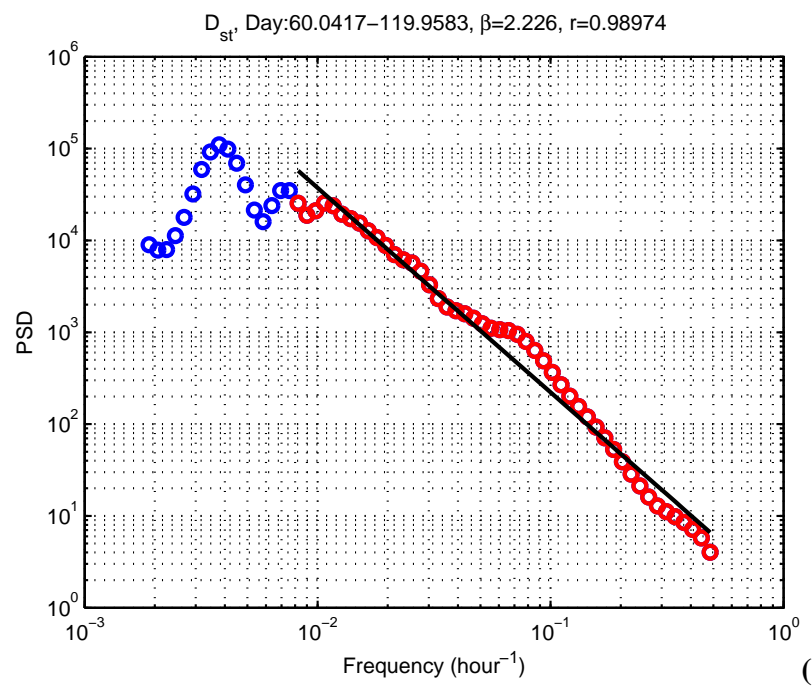

(b)

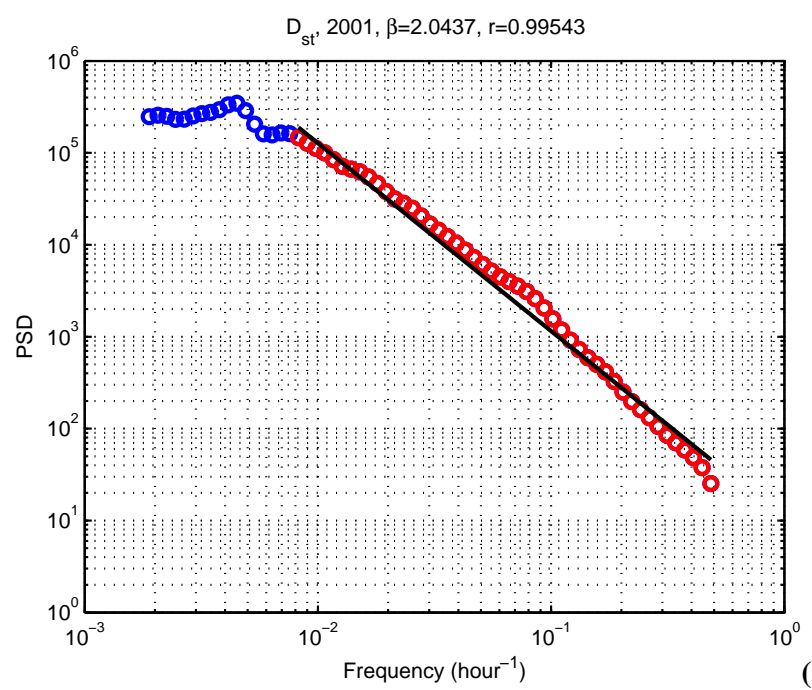

Fig. 5. The power spectral density functions of the $D_{s t}$ index for days 150-240 (a), 60-120 (b), 270-330 (c) and for all days in year 2001 (d). The red points correspond to periods $2-128 \mathrm{~h}$. The black line gives a fitted power-law of the form $S(f) \propto f^{-\beta}$ to these periods.

unity in some cases. On 31 March $2001 r$ becomes almost 1, whereas on 6 November 2001 it drops close to 0.8 . We also found that the $V B_{\text {South }}$ power spectrum either implies fGn behavior (epochs with $0<\beta<1$ ), or signifies an activity compatible with the fBm model possessing anti-persistent properties (epochs with $1<\beta<2$ ). Indeed, the exponent increases with activity, but never reaches the persistent regime like $D_{s t}$ does (c.f. $\beta_{V B_{\text {South }}}$ plot in Fig. 2). Thus, the transition in the $D_{s t}$ scaling, i.e. the transition from the anti-persistent to persistent regime, also reflects the effects of internal magnetospheric processes rather than interplanetary variations alone. This is consistent with studies showing that interplanetary driving through southward-oriented magnetic fields is not always by itself sufficient to drive intense MSs, because it is subject to modulation by internal magnetospheric conditions (Daglis et al., 2003).

A close up of the $V B_{\text {South }}$ time series from days 60 to 120 and 280 to 340 , as well as the corresponding wavelet power spectra and spectral parameters $r$ and $\beta$ are given in Figs. $4 \mathrm{c}-4 \mathrm{~d}$, respectively. Note that the wavelet spectra of $D_{s t}$ and $V B_{\text {South }}$ are remarkably similar around 31 March 2001 (Figs. 4a and 4c). Since the wavelet spectrum is essentially a power spectrum in the wavelet basis, the $D_{s t}$ scaling transition is due to the phase spectrum and its evolution in the course of the storm. For the other event (around 6 November 2001), the wavelet spectra are different for the two variables (Figs. $4 \mathrm{~b}$ and $4 \mathrm{~d}$ ). These differences enhance our point of view that some of the magnetospheric variability has inter- 
nal origin and it is not solely due to the solar wind conditions.

In Figs. 6a-6b $D_{s t}$ and $V B_{\text {South variations, as well as cor- }}$ responding $r$ and $\beta$ parameters from another year (1989), are shown. This year represents the previous solar cycle maximum. 1989 includes the largest magnetic storm ever recorded (-589 nT, 14 March 1989) and a group of 3 intense magnetic storms which occurred within a time interval of 3 months (-255 nT, 19 September 1989; -268 nT, 21 October $1989 ;-266 \mathrm{nT}, 17$ November 1989). Note that the $\beta$ values for $D_{s t}$ are well above $2(\beta>2.2)$ for a time interval of approximately one month in the first case and for almost 3 months in the second case, respectively (see red color in Fig. 6a), while $\beta$ never exceeds 1.5 for the $V B_{\text {South values }}$ (Fig. 6b).

\section{A possible scenario for the development of intense MSs}

Maslov et al. (1994) have formally established the relationship between spatial fractal behavior and long-range temporal correlations for a broad range of critical phenomena. By studying the time correlations in the local activity, they show that the temporal and spatial activity can be described as different cuts in the same underlying fractal. In a geometrical sense, $\beta$ specifies the strength of the signal irregularity as well. The fractal dimension $D$ is calculated from the relation

$D=(5-\beta) / 2$

for the fBm random field model (Heneghan and McDarby, 2000), which, after considering the shift of $\beta$ to higher values, leads to a decrease in the fractal dimension as the magnetic storm approaches. This may reflect that the action of anisotropy inherent to the system leads to the appearance of a clear preferred direction of elementary activities (ion acceleration and earthward transport) just before the main event of the system's evolution, which is the massive and continuous earthward injection of accelerated ions. Theoretical and experimental evidence support the former hypothesis: throughout the entire main phase and during the early recovery phase of intense MSs the geometry of the energy flow produces a highly asymmetric ring current configuration (Daglis and Kozyra, 2002; Daglis et al., 2003; Balasis et al., 2004; Balasis and Egbert, 2006). The emergence of strong anisotropy rationalizes a further reduction in complexity with time.

By monitoring the temporal evolution of the fractal spectral characteristics in $D_{s t}$ we found that distinct alterations in the associated scaling parameters indicate a transition from the low-activity state to an abnormal/high-activity state (intense MS) as follows: (i) emergence of long-range correlations, i.e. appearance of memory effects. This implies a multi-time-scale cooperative activity of numerous activated units. (ii) Increase in the spatial correlation in the time series with time. This indicates a gradual transition from a less orderly state to a more orderly state. (iii) Decrease in the

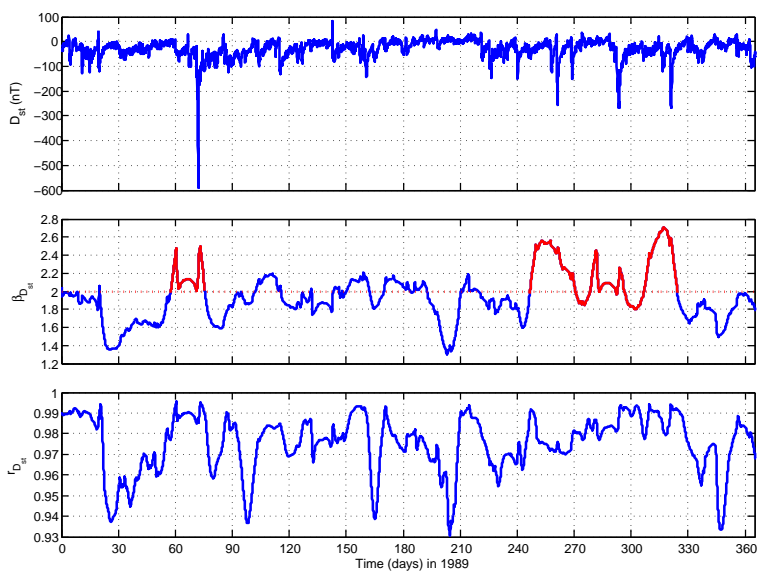

(a)
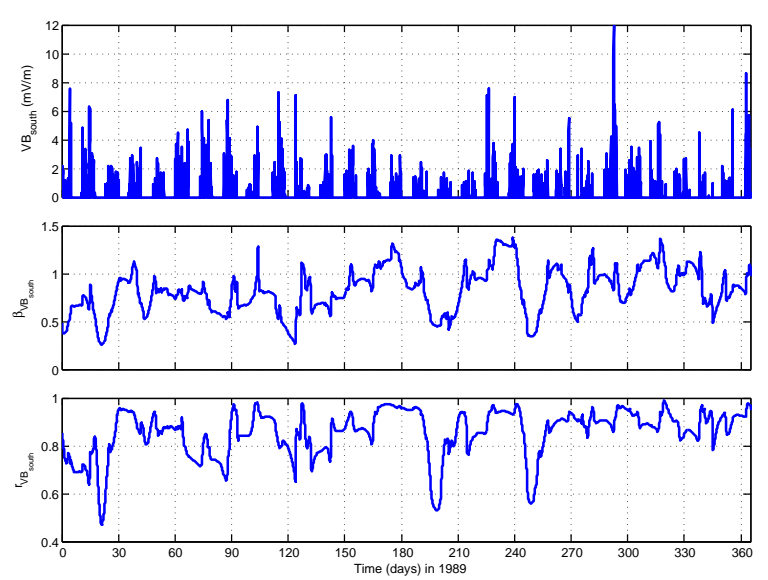

(b)

Fig. 6. (a-b) $D_{s t}$ or $V B_{\text {South }}$ time series, corresponding linear correlation coefficients $r$ and spectral exponents $\beta$. The red dashed line in $\beta D_{s t}$ plot (b) marks the transition between anti-persistent and persistent behavior.

fractal dimension of the variations with time, i.e. appearance of strong anisotropy in elementary activities. (iv) Existence of strong anti-persistent behavior in the pre-main phase interval, i.e. prior to the intense MS. (v) Decrease in the antipersistent behavior with time. (vi) Emergence of persistent properties in the tail of the time series. (vii) Predominance of large magnetospheric events with time. (viii) Significant acceleration of the energy release as the main event approaches, i.e. increase in the susceptibility of the system. (ix) Gradual appearance of higher frequencies in the spectrum with a simultaneous increase in the amplitudes at each emission rate as the MS peak approaches, mainly characterizing lower emission rates.

The aforementioned signatures (including temporal changes in scaling parameters) distinguish the dynamics of a complex system close to its instability. They may 
indicate the following scenario for the development of an intense MS. During the pre-storm period, the system is in a sub-critical anti-persistent state, with a restricted and systematically fluctuating correlation length. Long-range correlations gradually build up through local interactions until instabilities extend throughout the entire system. Such instabilities can channel the magnetosphere into a globally stable non-equilibrium super-critical state. Substorms may be the agents by which longer correlations are established. A population of substorms, as a result of the solar wind-magnetosphere coupling, will advance the correlation length by an amount depending on the coupling magnitude and duration and on the magnetosphere state, triggering intense MSs only if the condition is right: in a sub-critical anti-persistent regime, a population of small solar wind energy inputs leads to a quickly decaying activity, namely substorms or weak storms which do not lead to massive ring current build-up. In the super-critical persistent state this process is just able to continue indefinitely. This explains in a natural way the following: when the magnetosphere is not close to criticality it can only exhibit a small response to a small external perturbation, whereas when the magnetosphere is close to criticality its response may become explosive to the same small external perturbation due to the high susceptibility of its system. An intense MS destroys long correlations, creating a new low-activity period during which the process repeats under the influence of the solar wind by rebuilding correlation lengths toward criticality and the next large event. Thus, a large shock of the system may act as a sort of critical point (Chang, 1992), dividing the geomagnetic cycle into a period of growing correlations before the great event and a relatively uncorrelated phase thereafter.

In terms of criticality the acceleration of activity is the consequence of the growth of the spatial correlation length. Thus, a large MS which is not immediately preceded by a period of accelerating activity may represent a system which had previously reached a critical state but has not yet had an MS large enough to perturb the system away from the critical state. On the other hand, an accelerating geomagnetic activity which is not followed by a large MS may mirror a system which has achieved criticality but in which a large event has not nucleated.

\section{Self-organized or intermittent criticality?}

The analogies with the dynamics of the SOC model for magnetospheric substorms have been discussed by numerous authors (Sitnov et al., 2001; Uritsky et al., 2002 and references therein). Pure SOC models imply a system perpetually near instability, hence reducing the degree of predictability of individual events. Our analysis suggests that the magnetosphere may not be in a state of continuous SOC. On the contrary, the aforementioned evolution incorporates a time dynamics with memory effects, which may be characterized overall as "intermittent criticality" (Bowman et al., 1998; Sammis and Sornette, 2002). Intermittent criticality predicts a time-dependent variation in the activity as the "critical point" is approached, implying, in contrast to SOC, a degree of predictability. The central prediction of this hypothesis is that large events occur when the system is near a critical state that is characterized by both extreme susceptibility to external factors and strong correlations between different activated parts of the systems (Bowman et al., 1998; Sammis and Sornette, 2002).

The present study suggests that it is important to distinguish between SOC and intermittent criticality in the study of the MS cycle. A proper recognition and understanding of tuning parameters may lead to improved magnetospheric models having higher performance reliability. One of the main features in the complexity is the role that the topological disorder plays in such systems. The range of size scales characterizing heterogeneities of the thresholds might act as a tuning parameter of the underlying final MS dynamics.

A question that arises is whether the evolution toward an instability of the system is inevitable after the appearance of distinct symptoms in the geomagnetic variations. The emergence of persistent behavior, the significant acceleration of the energy release, i.e. the increase in the susceptibility of the system, the appearance of fluctuations at all scales with a simultaneous predominance of large geomagnetic events, the emergence of strong anisotropy, namely the appearance of preferential direction, indicate that the generation of an intense MS becomes, indeed, unavoidable.

\section{On the transition from the anti-persistent to persis- tent regime}

We focus on the observed interesting transition from the antipersistent to the persistent regime. The anti-persistent behavior characterizes the magnetosphere during substorms, while the catastrophic events are in reasonable agreement with persistent models. Recent studies have also underlined the existence of two different regimes in agreement with our proposal. We note that Sitnov et al. (2001) have suggested that while substorm activity resembles second-order phase transitions, the largest substorm avalanches (occurring during intense MSs) are shown to reveal features of first-order non-equilibrium transitions. Although there is no evidence of energy storage or turbulence in the magnetic topology of the inner magnetosphere the corresponding pictures in each regime (anti-persistent/second-order phase transitions - persistent/first order non-equilibrium transitions) are not in contradiction.

The point of view that the substorm activity resembles the nonequilibrium (first and/or second order) phase transition (Sitnov et al., 2001) is also consistent with the "magnetic topological complexity" emerging from Chang's model 
(Chang, 1999, 2001a). In the frame of this approach, if we consider a physical system with a given disorder magnetic field structure, the minimum free energy is a function of the topological complexity of the magnetic field. In such a system, any non-ideal process which modifies the overall topology may be associated with a sort of a dynamical transition between two different local minima in the configuration space during which a certain amount of free energy is relaxed. The emerging dynamical framework is that of a random walk in a complex free energy landscape. If the system evolves near criticality, this random walk in the free energy space will be characterized by a time correlation in the jumps. In this framework, magnetic substorms are better described in terms of noise-induced topological transitions in an extended out-of-equilibrium system. In other words, the magnetic substorm is the set of phenomena during which a reduction in topological complexity takes place (Chang, 2001a, 2001b; Consolini and Chang, 2001, 2002; Consolini and De Michelis, 2002). The role of the solar wind driver would be to enhance the internal noise (i.e. the internal fluctuations) which could induce a topological transition among metastable complex topologies. In such a case, the evolution of the magnetospheric system will be the result of the combined effects of local couplings of the magnetic and plasmas structures, and of the noise intensity through the nonlinearities of the system. The aforementioned evolutionary scenario is one of strong dynamics, and involves the evolution among first-order and second-order transitions of topological complexity (Consolini and Chang, 2001). Furthermore, Chang et al. $(2003,2004)$ described a theory of intermittent turbulence in space plasmas which agrees with the idea of intermittent criticality discussed in the previous section.

\section{Conclusions}

The fractal spectral analysis applied here indicates that intense MSs are not clear SOC phenomena. SOC implies a system perpetually near global failure. This suggests large correlations would be maintained in the system, hence reducing the degree of predictability of individual intense MSs. However, several results of the present analysis have been interpreted in the frame of the intermittent criticality approach (Bowman et al., 1998; Sammis and Sornette, 2002), implying instead a magnetosphere maintained predominantly in a subcritical state, with fluctuations during the intense MS representing super-critical state. The preparation of an intense MS in the frame of the intermittent criticality, that is of a more general character than the classical SOC phenomena (Bak et al., 1987), implies a degree of predictability in the population dynamics (Sornette, 2004). Our results are consistent with the emergence of intermittent criticality.

Our analysis suggests as a main result that a spectral signature related to the emergence of persistency of the $D_{s t}$ time series precedes an intense MS. The spectral signature is inter- preted as a critical increase in the susceptibility of the system, an effect typical for a system in the super-critical state, where any small instabilities can provoke large-scale reactions accompanied by essential energy release.

Recently, Chang et al. (2006) have noted the following: "turbulence in space plasmas generally encompass fluctuations of all varieties and sizes, which interact and propagate throughout the plasma medium: multitudes of different sizes of large scale (i.e. sizes much larger than the original particle sizes of the plasma medium) 'coherent structures' are formed. When coherent magnetic flux tubes with the same polarity migrate toward each other, strong local magnetic shears are created. It has been demonstrated that existing sporadic nonpropagating fluctuations will generally migrate toward the strong local magnetic shear region. Eventually the mean local energies of the coherent structures will be dissipated into these concentrated fluctuations in the coarsegrained sense and, thereby reconfigure the topologies of the coherent structures of the same polarity into a combined lower local energetic state, eventually allowing the coherent structures to merge locally. And, this merging or 'magnetic reconfiguration' process may repeat over and over again among the coherent structures. The fluctuations that are induced by such processes are sporadic and localized. Because the coherent structures are numerous and outsized, we expect that the fluctuations within the interaction regions of these structures (resonance overlap regions) are generally large and can occur relatively often compared to those that would have been expected from a medium of uniformly sized plasma particles. Such statistical characteristic of 'extreme events' exemplifies the phenomenon of intermittent turbulence in space plasmas". The whole description, perhaps not surprisingly, seems to fit to our magnetospheric evolution scenario presented in Sect. 4: the creation of the strong local magnetic shears agrees with the appearance of a clear preferred direction of elementary activities; the magnetic reconfiguration process may be repeated over and over again among the coherent structures, as in the persistent state the local interactions are just able to continue indefinitely etc.

Two lines of evidence support one of the main results of our analysis: while the magnetosphere is mostly driven by the solar wind the critical feature of persistency in the magnetosphere is the result of a combination of solar wind and internal magnetospheric activity rather than solar wind variations alone. First, a search for a spectral signature in the main interplanetary driver of $D_{s t}$, the $V B_{\text {South }}$ electric field component, does not reveal a similar transition to persistency prior to or around the storm. Second, the wavelet spectra of $D_{s t}$ and $V B_{\text {South }}$ are remarkably similar around 31 March 2001 but quite different, around 6 November 2001, with the $D_{s t}$ spectrum possessing more power. These differences enhance our point of view that some of the magnetospheric variability has internal origin and is not solely due to the solar wind conditions. This proposal is in agreement with the suggestion that the system may evolve toward a sort 
of dynamical critical state, where a hierarchy of scale-free coherent structures can be generated from the macroscopic fluctuations of the system itself (Consolini and Chang, 2001).

Other studies also indicate the existence of two different regimes in the dynamics of magnetosphere. Sitnov et al. (2001) suggest that the substorm dynamics resembles second-order phase transitions, while MSs, are shown to reveal the features of first-order non-equilibrium transitions. The anti-persistency/persistency meet well the second order/first order phase transition correspondingly. Metastability and topological complexity of the magnetic field, emerging from Chang's model (1999), also justify the transition under study. Results from the analysis of data from another year (1989) support the findings reported in the previous paragraph.

As it is noticed, a basic reason for our interest in complexity is the striking similarity in behavior near critical point among systems that are otherwise quite different in nature (Stanley, 1999, 2000; Viczek, 2001, 2002; Sornette, 2004). The above-mentioned picture of magnetospheric dynamics in response to solar wind is quite similar to what is observed in a different framework (Consolini and Chang, 2002): the origin of the biological function in biochemistry (Careri, 1982, 1998). Enzymatic activity is indeed due to strong correlations among the various spontaneous fluctuations. We underline that the herein found distinct alterations as an intense MS approaches (e.g. transition from anti-persistency to persistency) were previously observed in the cases of seismic shocks and epileptic seizures (Kapiris et al., 2005), and thus imply a degree of universality in the behavior of these extreme events around their final stage.

For some years now there has been a mild controversy ( $\mathrm{Li}$ et al., 2003) simmering between those who emphasize that the magnetosphere is mostly driven by the solar wind and thus its variability is due to the variability of the solar wind, and those who emphasize that some of its variability is internally generated. Recently, Temerin and Li (2002) suggested that global magnetospheric features are driven by the solar wind and are predictable in a rather deterministic sense. For such features, chaotic behavior within the magnetosphere has little influence on the global outcome (Li et al., 2003). However, as Li et al. (2003) noted, the degree to which the magnetotail may exhibit turbulent behavior independent of the solar wind and thus influence the exact timing of substorms, recurrent smaller-scale enhancements in magnetic activity, is not yet known and highly controversial. The results of the present study suggest that all of these concepts remain to be investigated further. As for future work, it would also be interesting for a comparison with the present results to extend our analysis to 1-minute SYM-H data.
Acknowledgements. G. Balasis acknowledges support from the Greek General Secretariat for Research and Technology under research grant ENTER 04ER/35.

The Editor-in-Chief W. Kofman thanks two referees for their help in evaluating this paper.

\section{References}

Akay, M.: Time Frequency and Wavelets in Biomedical Signal Processing Engineering, Wiley-IEEE Press, pp. 768, 1997.

Amaral, L., Goldberger, A., Ivanov, P., and Stanley, H.: ScaleIndependent Measures and Pathologic Cardiac Dynamics, Phys. Rev. Lett., 81, 2388-2391, 1998.

Bak, P.: How nature works, Springer, New York, 212 pp., 1996.

Bak, P., Tang, C., and Wiesenfeld, K.: Self-organized criticality: an explanation of 1/f noise, Phys. Rev. Lett., 59, 381-383, 1987.

Balasis, G., Egbert, G. D., and Maus, S.: Local time effects in satellite estimates of electromagnetic induction transfer functions, Geophys. Res. Lett., 31, doi:10.1029/2004GL020147, 2004.

Balasis, G., Maus, S., Lühr, H., and Rother, M.: Wavelet analysis of CHAMP flux gate magnetometer data, in: Earth Observation with CHAMP, edited by: Reigber, C., Lühr, H., Schwintzer, P., and Wickert, J., Springer, New York, 347-352, 2005.

Balasis, G. and Egbert, G. D.: Empirical orthogonal function analysis of magnetic observatory data: Further evidence for non-axisymmetric magnetospheric sources for satellite induction studies, Geophys. Res. Lett., 33, doi:10.1029/2006GL025721, 2006.

Bowman, D. D., Ouillon, G., Sammis, C. G., Sornette, A., and Sornette, D.: An observational test of the critical earthquake concept, J. Geophys. Res., 103, 24359-24372, 1998.

Careri, G.: Ordine e disordine nella materia, Laterza, Roma, 1982.

Careri, G.: Cooperative charge fluctuations by migrating protons in globular proteins, Progress in Biophysics and Molecular Biology, 70, 223-249, 1998.

Chang, T.: Low-dimensional behavior and symmetry-breaking of stochastic systems near criticality - can these effects be observed in space and in the laboratory?, IEEE Trans. Plasma Sci., 20, 6, 691-694, 1992.

Chang, T.: Self-organized criticality, multi-fractal spectra, sporadic localized reconnections and intermittent turbulence in magnetotail, Phys. Plasmas, 6, 4137-4145, 1999.

Chang, T.: An example of resonances, coherent structures and topological phase transition - the origin of the low frequency broadband spectrum in the auroral zone, Nonlinear Proc. Geophys., 8 , 175-180, 2001a.

Chang, T.: Colloid-like behavior and topological phase transitions in space plasmas: intermittent low frequency turbulence in the auroral zone, Phys. Scripta, T89, 80-83, 2001b.

Chang, T., Tam, S. W. Y., Wu, C. C., and Consolini G.: Complexity, Forced and/or Self-Organized Criticality, and Topological Phase Transitions in Space Plasmas, Space Science Reviews, 107, 425445, 2003.

Chang, T., Tam, S. W. Y., and Wu, C. C.: Complexity Induced Anisotropic Bimodal Intermittent Turbulence in Space Plasmas, Physics of Plasmas, 11, 1287-1299, 2004.

Chang, T., Tam, S. W. Y., and Wu, C. C.: Complexity in Space Plasmas - A Brief Review, Space Science Reviews, 122, 281291, doi:10.1007/s11214-006-5957-4, 2006. 
Consolini, G., and Chang, T.: Magnetic field topology and criticality in geotail dynamics: relevance to substorm phenomena, Space Sci. Rev., 95, 309-321, 2001.

Consolini. G. and De Michelis P.: Fractal time statistics of AEindex burst waiting times: evidence of metastability, Nonlinear Process. in Geophys., 9, 419-423, 2002.

Consolini, G. and Chang, T.: Complexity, magnetic field topology, criticality, and metastability in magnetotail dynamics, J. Atmos. Sol. Terr. Phys., 64, 541-549, 2002.

Daglis, I. A., Baker, D. N, Galperin, Y., Kappenman, J. G., and Lanzerotti, L. J.: Technological impacts of space storms: Outstanding issues, Eos Trans. AGU, 82, 585, doi:10.1029/01EO00340, 2001.

Daglis, I. A. and Kozyra, J. U.: Outstanding issues of ring current dynamics, J. Atmos. Solar-Terr. Phys., 64, 253-264, 2002.

Daglis, I. A., Kozyra, J. U., Kamide, Y., Vassiliadis, D., Sharma, A. S., Liemohn, M. W., Gonzalez, W. D., Tsurutani, B. T., and Lu, G.: Intense space storms: Critical issues and open disputes, J. Geophys. Res., 108, 1208, doi:10.1029/2002JA009722, 2003.

Daglis, I. A., Delcourt, D., Metallinou, F.-A., and Kamide, Y.: Particle acceleration in the frame of the storm-substorm relation, IEEE Trans. Plasma Science, 32, 1449-1454, 2004.

Freeman, M. P. and Watkins, N. W.: The Heavens in a Pile of Sand, Science, 298, 979-980, 2002.

Gedalin, M., Balikhin, M., Coca, D., Consolini, G., and Treumann R. A.: Kinetic description of avalanching systems, Phys. Rev. E, 72, doi:10.1103/PhysRevE.72.037103, 2005.

Gilden, D., Thornton, T., and Mallon, M.: 1/f noise in human cognition, Science, 267, 1837-1839, 1995.

Heneghan, C. and McDarby, G.: Establishing the relation between detrended fluctuation analysis and power spectral density analysis for stochastic processes, Phys. Rev. E., 62, 6103-6110, 2000.

Jun, Y. and $\mathrm{Wu}, \mathrm{X}$. L.: Large-scale intermittency in two-dimensional driven turbulence, Phys. Rev. E, 72, doi:10.1103/PhysRevE.72.035302, 2005.

Kapiris, P., Eftaxias, K., Nomikos, K., Polygiannakis, J., Dologlou, E., Balasis, G., Bogris, N., Peratzakis, A., and Hadjikontis, V.: Evolving towards a critical point: A possible electromagnetic way in which the critical regime is reached as the rupture approaches, Nonl. Proc. Geophys., 10, 511-524, 2003.

Kapiris, P., Balasis, G., Kopanas, J., Antonopoulos, G., Peratzakis, A., and Eftaxias, K.: Scaling similarities of multiple fracturing in solid materials, Nonl. Proc. Geophys., 11, 137-151, 2004a.

Kapiris, P., Eftaxias, K., and Chelidze, T.: Electromagnetic Signature of Prefracture Criticality in Heterogeneous Media, Phys. Rev. Lett., 92, doi:10.1103/PhysRevLett.92.065702, 2004b.

Kapiris, P., Polygiannakis, J., Li, X., Yao, X., and Eftaxias, K.: Similarities in precursory features in seismic shocks and epileptic seizures, Europhys. Lett., 69, 657-663, 2005.

Kiyono, K., Struzik, Z. R., and Yamamoto, Y.: Criticality and Phase Transition in Stock-Price Fluctuations, Phys. Rev. Lett., 96, doi:10.1103/PhysRevLett.96.068701, 2006.

Kumar, P., and Foufoula-Georgiou, E.: Wavelet analysis for geophysical applications, Rev. Geophys., 35, 385-412, 1997.

Li, X., Temerin, M., Baker, D. N., Reeves, G. D., Larson, D., and Kanekal, S. G.: The Predictability of the Magnetosphere and Space Weather, EOS Transactions, 84, doi:10.1029/2003EO370002, 2003.
Liemohn, M. and Kozyra, J. U.: Lognormal form of the ring-current energy content, J. Atm. Sol.-Terr. Phys., 65, 871-886, 2003.

Malamud, B., Morein, G., and Turcotte, D.: Forest fires: an example of self-oganized critical behavior, Science, 281, 1840-1842, 1998.

Mandea, M. and Balasis, G.: The SGR 1806-20 magnetar signature on the Earth's magnetic field, doi:10.1111/j.1365246X.2006.03125.x, 2006.

Mandelbrot, B. and Ness, J., Fractional Brownian motions, fractional noises and applications, SIAM Rev., 10, 422-437, 1968.

Mandelbrot, B.: Fractals and Scaling in Finance, Springer, New York, 551 pp., 2005.

Maslov, S., Paczuski, M., and Bak, P.: Avalanches and 1/f Noise in Evolution and Growth Models, Phys. Rev. Lett., 73, 2162-2165, 1994.

Peng, C., Havlin S., Stanley, H., and Goldberger, A.: Quantification of scaling exponents and crossover phenomena in nonstationary heartbeat time series, Chaos, 5, 82-87, 1995.

Sammis, C. G., and Sornette, D.: Positive feedback, memory, and the predictability of earthquakes, Proc. Nat. Acad. Sci., 99, 2501-2508, 2002.

Sitnov, M. I., Sharma, A. S., Papadopoulos, K., and Vassiliadis, D.: Modeling substorm dynamics of the magnetosphere: From self-organization and self-organized criticality to nonequilibrium phase transitions, Phys. Rev. E, 65, doi:10.1103/PhysRevE.65.016116, 2001.

Sornette, D.: Critical Phenomena in Natural Sciences, Springer, Heidelberg, 528 pp., 2004.

Stanley, H. E.: Scaling, universality, and renormalization: Three pillars of modern critical phenomena, Rev. Mod. Phys., 71, S358-S366, 1999.

Stanley, H.: Exotic statistical physics: Applications to biology, medicine, and economics, Physica A, 285, 1-17, 2000.

Takalo, J., Lohikosiki, R., and Timonen, J.: Structure function as a tool in AE and Dst time series analysis, Geophys. Res. Lett., 22, 635-638, 1995.

Temerin, M. and Li, X.: A new model for the prediction of Dst on the basis of the solar wind, J. Geophys. Res., 107, A12, 1472, doi:10.1029/2001JA007532, 2002.

Torrence, C. and Compo, G. P.: A Practical Guide to Wavelet Analysis, Bull. Am. Meteor. Soc., 79, 61-78, 1998.

Turcotte, D. L.: Fractals and Chaos in Geology and Geophysics, Cambridge Univ. Press, 398 pp., 1997.

Uritsky, V. M., Klimas, A. J., Vassiliadis, D., Chua, D., and Parks, G.: Scale-free statistics of spatiotemporal auroral emissions as depicted by POLAR UVI images: Dynamic magnetosphere is an avalanching system, J. Geophys. Res., 107, (A12), doi:10.1029/2001JA000281, 2002.

Vicsek, T.: A question of scale, Nature, 411, 421, 2001.

Vicsek, T.: The bigger picture, Nature, 418, 131, 2002.

Wei, H. L., Billings, S. A., and Balikhin, M.: Analysis of the geomagnetic activity of the Dst index and self affine fractals using wavelet transforms, Nonlinear Process. in Geophys., 11, 303312, 2004.

Worrell, G., Cranstoun, S., Litt, B., and Echauz, J.: Evidence for self-organized criticality in human epileptic hippocampus, Neurophysiology, Basic and Clinical, 13(16), 2017-2021, 2002. 